\title{
Combining Virtual Reality and Behavioral Health to Promote Pain Resiliency: Analysis of a Novel BioPsychoSocial Modality for Solving Pain in the Workplace
}

\author{
Alaa Abd-Elsayed · Nasir Hussain · Gerry Stanley
}

Received: August 10, 2021 / Accepted: September 28, 2021 / Published online: October 16, 2021

(C) The Author(s) 2021

\begin{abstract}
Background: The use of virtual reality in treating pain symptoms is a new and emerging modality that has gained more traction, especially in light of the COVID-19 pandemic, which has led to a rise in the remote care of patients. Our study tested the effectiveness of a home-based pain relief program that utilized virtual reality and behavioral health for treating acute and chronic pain.
\end{abstract}

Study Design: Retrospective Cohort Study.

Methods: Due to the remote nature of the pain clinic during the COVID-19 pandemic, patients with work-related injuries were considered for inclusion in a 90-day Harvard MedTech Vx Pain Relief Program, which consisted of home-based virtual reality therapy (VRT) and tailored behavioral therapy that was conducted through phone consultations. Both the VRT and

\footnotetext{
A. Abd-Elsayed ( $\square)$

Department of Anesthesiology, School of Medicine and Public Health, University of Wisconsin, 600

Highland Avenue, B6/319 CSC, Madison,

WI 53792-3272, USA

e-mail: alaaawny@hotmail.com

N. Hussain

Department of Pain Medicine, Beth Israel Deaconess

Medical Center, Harvard Medical Center, Boston,

MA, USA

G. Stanley

Harvard MEDTECH, Las Vegas, NV, USA
}

behavioral therapy focused on the specific workplace injury and centered around setting three personal goals for each patient that were considered meaningful to them. The progress towards achieving these goals was then tracked. The primary outcome of the retrospective analysis was pain scores after completion of home-based VRT. Secondary outcomes included: change in opioid therapy (opioid consumption), psychological awareness/perception of pain, level of immersion, and overall improvement/achievement in patient goals.

Results: A total of 36 patients (16 male and 20 female) with acute and chronic pain secondary to workplace injuries were included; these injuries severely limited their activities of daily living and reduced their quality of life. During the course of VRT and after completion of the 90-day program, patients experienced a substantial immediate and legacy pain relief. Specifically, patients reported an average $40 \%$ reduction in pain while utilizing the program and an additional $2.8 \mathrm{~h}$ of continued pain relief at the conclusion of 90 days. Further, $69 \%$ of patients also reported either a decrease in opioid use or complete cessation after VRT. The majority of patients also reported reduced depression, fatigue, and sleep disturbance.

Conclusions: The Harvard MedTech Vx Therapy appears to be an effective tool in the treatment of pain and the psycho-social issues associated with pain. This translates into a greater degree of pain resiliency and an overall 
improvement in general wellness and improved workplace functionality.

Keywords: Chronic pain; Virtual reality; Workers compensation; Pain psychology

\section{Key Summary Points}

Virtual reality is an emerging modality for treating chronic pain.

Our study tested the use of a virtual reality program for treating chronic pain in patients with work-related injuries.

Our results showed efficacy of virtual reality in improving chronic pain.

Virtual reality adds another tool to treat pain and can provide an alternative to opioids.

\section{INTRODUCTION}

With the onset of the 2019 novel coronavirus (COVID-19) pandemic, there was a rapid shift towards caring for patients virtually through telemedicine [1]. Although the clinical outcomes of such a shift in practice were not well described, this shift presented a unique opportunity to improve the care of patients through remote care. For pain patients specifically, where treating mental health and psychological well-being is equally as important as the physical state of pain itself, [2] the COVID-19 pandemic introduced novel virtual treatment avenues for the physician. Indeed, evidence has indicated that virtual mental-health counseling can be equally as effective as in-person patient visits [3].

With the transition to virtual clinics, physicians were tasked with identifying means to remotely treat both acute and chronic pain states. Virtual reality is one such means that could be considered as a part of the toolbox and is thought to improve pain through multimodal sensory distractions [4]. Originally only considered for entertainment value, the COVID-19 pandemic provided the opportunity to implement this technology for the treatment of pain and psychiatric disorders [4]. However, the true benefits of using such technology in improving the overall pain state, including pain psychology/perception, the behavioral aspects of pain, and opioid consumption still remains largely unknown.

The Harvard MedTech Vx Pain Relief Program is one such new technology that leverages home-based virtual reality with behavioral health therapy to help treat acute and chronic pain states. Although one aim of the program is to improve pain, the ultimate goal is to provide lasting relief along with the behavioral changes needed to get the patient back to work and restore a sense of normalcy to their social life. With the COVID-19 pandemic in hand, this created an opportunity to evaluate the impact of using such a technology for patient care.

Thus, the aim of this retrospective cohort study was to evaluate the effectiveness of the Harvard MedTech Vx Pain Relief Program on adult patients with acute and chronic pain. Specifically, our primary outcome was to assess the impact of such therapy on improving overall pain scores. The secondary aims were to evaluate its use on opioid consumption, pain perception, and the behavioral aspects of pain.

\section{METHODS}

In the setting of the COVID-19 pandemic, this retrospective cohort study received institutional review board (IRB) exempt status due to investigation of a benign behavioral intervention. This study was performed in accordance with the Helsinki Declaration of 1964 and its later amendments.

\section{Study Sample and Setting}

All adult patients aged 18 to 65 treated from April 2019 to April 2020 for acute or chronic workplace injuries and were on workers' compensation were retrospectively identified for inclusion. All patients were referred to and overseen by a prescribing physician and referred 
to the Harvard MedTech Vx Pain Relief Program by orthopedic specialists, pain specialists, primary care physicians, and occupational health providers. All types of workplace injuries were considered, and patients were excluded if their attorney required them to discontinue therapy. Patients were also excluded if they attended less than five in-person behavioral therapy sessions with their behavioral health clinician, as this would indicate possible poor compliance with the Harvard MedTech Vx Pain Relief Program.

\section{Study Intervention/Procedure}

The Harvard MedTech Vx Pain Relief Program consisted of both home-based virtual reality therapy (VRT) and tailored behavioral therapy that was conducted through phone consultations. The length of therapy lasted 90 days, or approximately 12 weeks. The VRT component of the program involved using the $\mathrm{Vx}$ headset (Manufactured by PICO), which patients were instructed to use at least 1 to 2 times daily for $45 \mathrm{~min}$. The headset was received by patients via mail after enrolling in the study and the patients would be contacted within 2 days to have their baseline phone call. During this first phone call, patients were instructed on how to use the headset and were asked a series of questions related to the study outcomes (discussed below) for baseline measurements. During headset use, patients selected specialized categories, or experiences, pre-loaded onto the headset to target either the knowledge of pain, meditation techniques, escape from pain, or distraction from pain. The choice to view a specific category of VRT was based on weekly phone calls between the physician and patient. For the tailored behavioral therapy component of the program, patients and clinicians discussed their progress with headset use (all outcomes re-reassessed) and the goals of therapy for 30-60 min each week and set three specific goals for the patients care. The goals could be related to pain reduction, sleep improvement, behavioral health, or social well-being. Any adjustments in therapy were also made at this meeting (as previously discussed). The patient's clinician directed the program, ensured compliance, and also provided further education on how to generalize the skills acquired by the virtual reality headset.

\section{Study Outcomes}

The primary outcome of this study was defined to be average pain relief, as defined on a Visual Analog Scale (VAS) score on $0-10(0=$ no pain, $10=$ worst pain imaginable) while actively using the Harvard MedTech Vx Pain Relief Program.

The secondary outcomes of this study included (1) change in opioid use; (2) average time thinking about pain; (3) anxiety awareness and level of immersion in therapy; (4) and patients' perception of set goals being achieved.

\section{Measurement of Outcome Data}

Pain was assessed using a 0-to-10-point VAS scale score using the Patient-Reported Outcomes Measurement Information System (PROMIS), with 0 representing no pain and 10 representing the worst pain imaginable. Similarly, perceived immersion in therapy and benefit of therapy was assessed using a 0-to-10point VAS scale score, with 0 representing no immersion/benefit and 10 representing maximal immersion or benefit. Opioid change data were represented as the proportion of patients either reducing, increasing, having no change, or stopping opioid use as a result of therapy. Average time thinking about pain was recorded in units of hours. Finally, in regard to assessing degree of progress towards patient-centered goals (discussed previously), metrics studied were whether or not the goals were met as well as the percentage of change towards specific goals.

The following questionnaires were also used to evaluate different component of each outcome previously discussed, including the Social Avoidance and Distress Scale (SADS) for depression and anxiety screening; the Pittsburgh Sleep Study for sleep screening; and the Short Post-Traumatic Stress Disorder Rating Interview (SPRINT) for post-traumatic stress disorder (PTSD) screening). 


\section{Data Collection and Analysis}

Data were collected for all outcomes from the physician-patient interactions during the weekly behavioral phone calls and patient records. The data were entered into a Microsoft Excel spreadsheet and stored on an encrypted server. All continuous outcome data (i.e., VAS score data [pain and degree of immersion/benefit of therapy] and average time thinking about pain) was reported as mean of the overall population. In contrast, all categorical data (i.e., opioid change data and patient goal data) were presented as frequency numbers.

\section{Sample Size}

Given that this was a pilot retrospective study to assess the effectiveness of the Harvard MedTech Vx Pain Relief Program, a sample size/power analysis calculation was not undertaken.

\section{Data Management}

Data analyses were performed using SPSS Statistics Version 26.0. All data were provided in aggregate form with means. All tests for significance were two-tailed with a $p$ value threshold of 0.05 .

\section{RESULTS}

A total of 36 patients (16 male and 20 female) who had acute and chronic pain secondary to workplace injuries were included; these injuries severely limited their activities of daily living and reduced their quality of life. The mean age of patients was 45 , with an age range of 20 to 65 . The types of injuries the patients incurred were in the (1) head and neck (eight patients); (2) legs and feet (ten patients); (3) shoulders and arms (11 patients); (4) back (15 patients); or v) hips, buttocks, glutes, and groin region (five patients). All patients had workplace injuries and were on workers' compensation. The mean baseline pain score of patients prior to participating in the program was noted to be $5.71 \mathrm{~cm}$.

\section{Primary Outcome}

\section{Pain Severity After Therapy}

Throughout each of the 12 weeks of the program, patients were noted to have considerably reduced mean VAS pain scores at each week after their virtual reality therapy session (Fig. 1). On average, patients experienced a reduction in pain by approximately $40 \%$ while using the virtual reality headset, and this was found to be statistically significant $(p<0.05)$ at each weekly session. Initially, patients only experienced this pain relief for $1.9 \mathrm{~h}$; however, with completion of each weekly session, this was found to be prolonged to approximately $2.8 \mathrm{~h}$ once the 12 -week program was completed.

\section{Secondary Outcomes}

\section{Average Hours Thinking About Pain}

In combination with pain relief, patients also appeared to have a reduction in the mean daily time spent thinking about pain symptoms. Specifically, the average time spent thinking about pain symptoms decreased from $9.78 \mathrm{~h}$ at the start of the therapy to $2.76 \mathrm{~h}$ after completion of the 12-week program, representing a roughly $72 \%$ reduction (Fig. 2 ). Importantly, the reduction in time thought about pain symptoms was found to broadly improve with each successive week of therapy.

\section{Opioid Therapy Change}

Patient opioid use was assessed at each behavioral visit session and dose changes were recorded. Overall, $69 \%$ of patients $(n=25)$ reported either a decrease in opioid use or complete cessation of opioids as a result of virtual reality therapy. In contrast, only $6 \%$ of patients $(n=2)$ reported an increase in opioid use as a result of virtual reality therapy (Fig. 3).

\section{Achievement of Patient-Centered Goals}

Patient-centered goals were created at the beginning of each visit pertaining to pain reduction, sleep improvement, behavioral health, or social well-being. The improvement in pain was previously discussed; however, the 


\section{Vx Pain Relief}

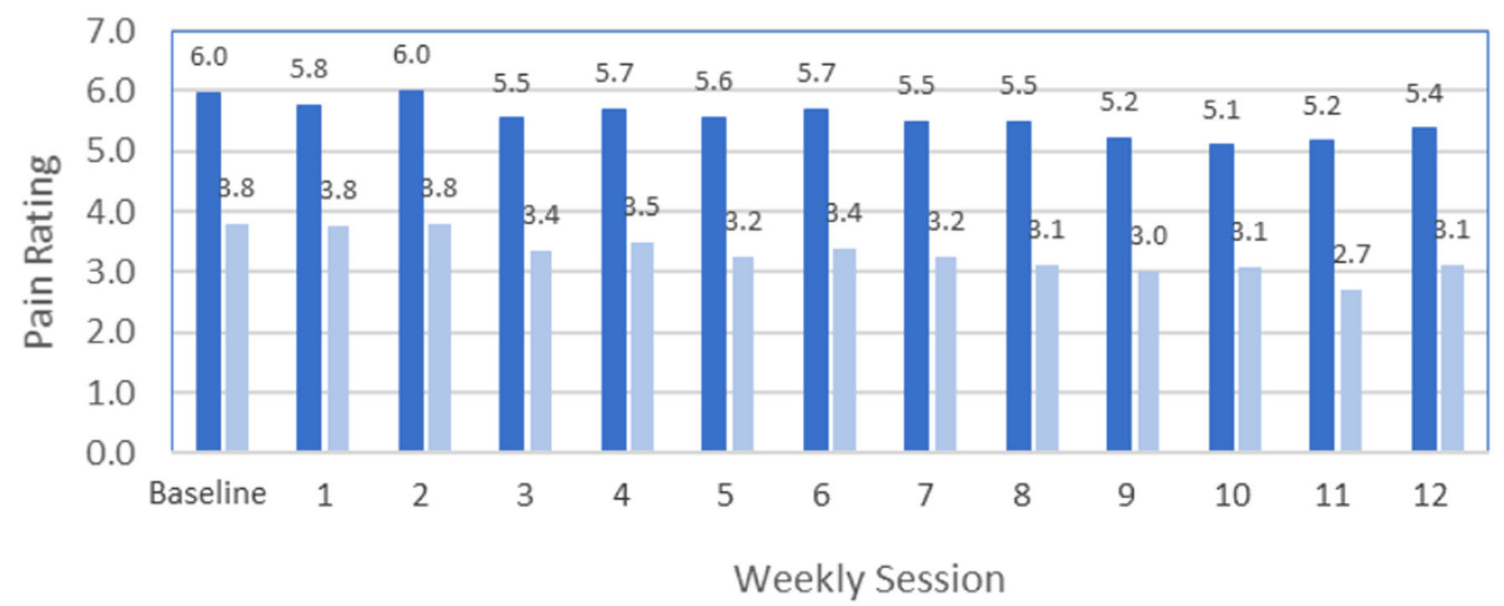

Pain Before Vx Therapy

Pain Following Vx Therapy

Fig. 1 Pain relief on a $0-10 \mathrm{~cm}$ visual analog scale (VAS) across each week before and after completion of virtual reality therapy. Baseline therapy represents the initial telephone encounter with patients when the headset was

first received, and prior to initiation of therapy. Values are represented as means

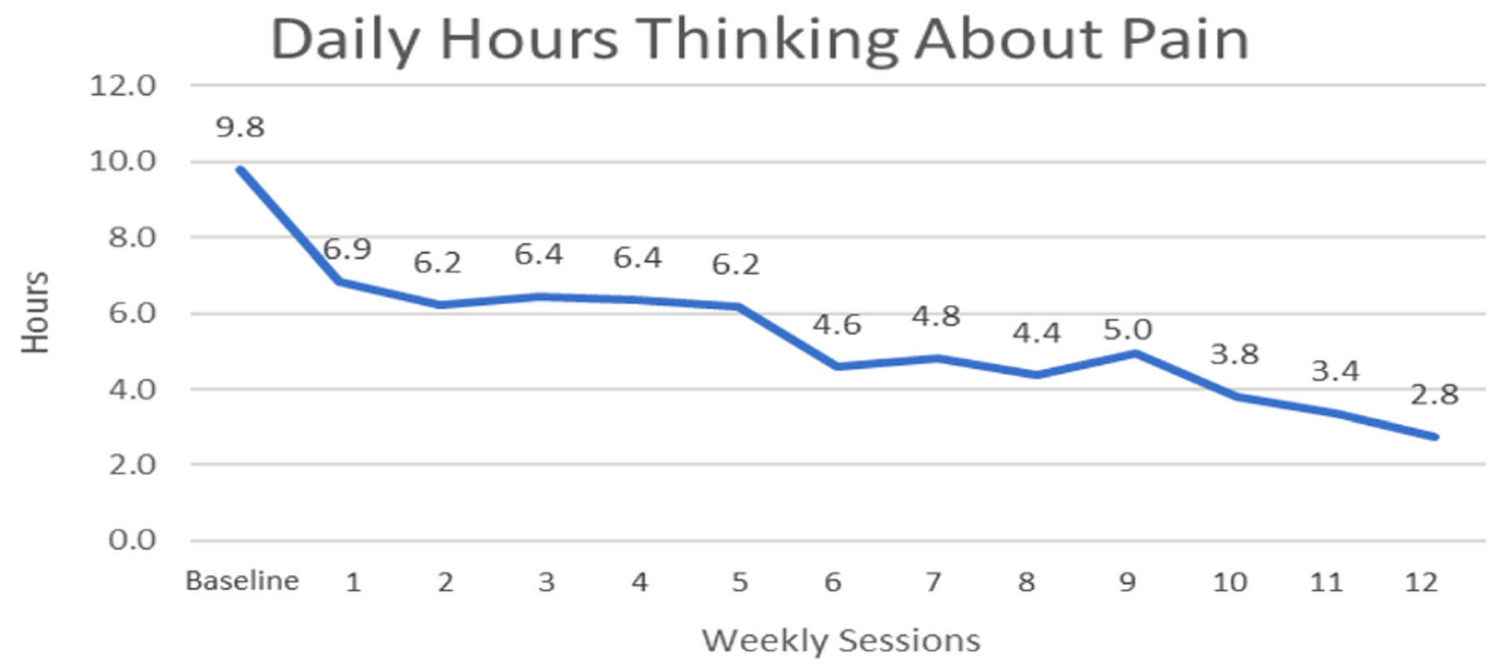

Fig. 2 Daily hours thinking about pain after each weekly session. Values are represented as means

remaining goal results are presented below (Fig. 4).

\section{Sleep Improvement}

Overall, patients experienced a $115 \%$ increase in the duration of their sleep and $280 \%$ increase in the subjective quality of their sleep after using virtual reality therapy. Importantly, patients reported an 85\% reduction in sleep interruptions. For those patients that experienced sleep interruptions, however, 92\% were able to fall back asleep more quickly after enrolling in the 12-week program (Fig. 5). 


\section{Opiate Change with Vx Therapy}

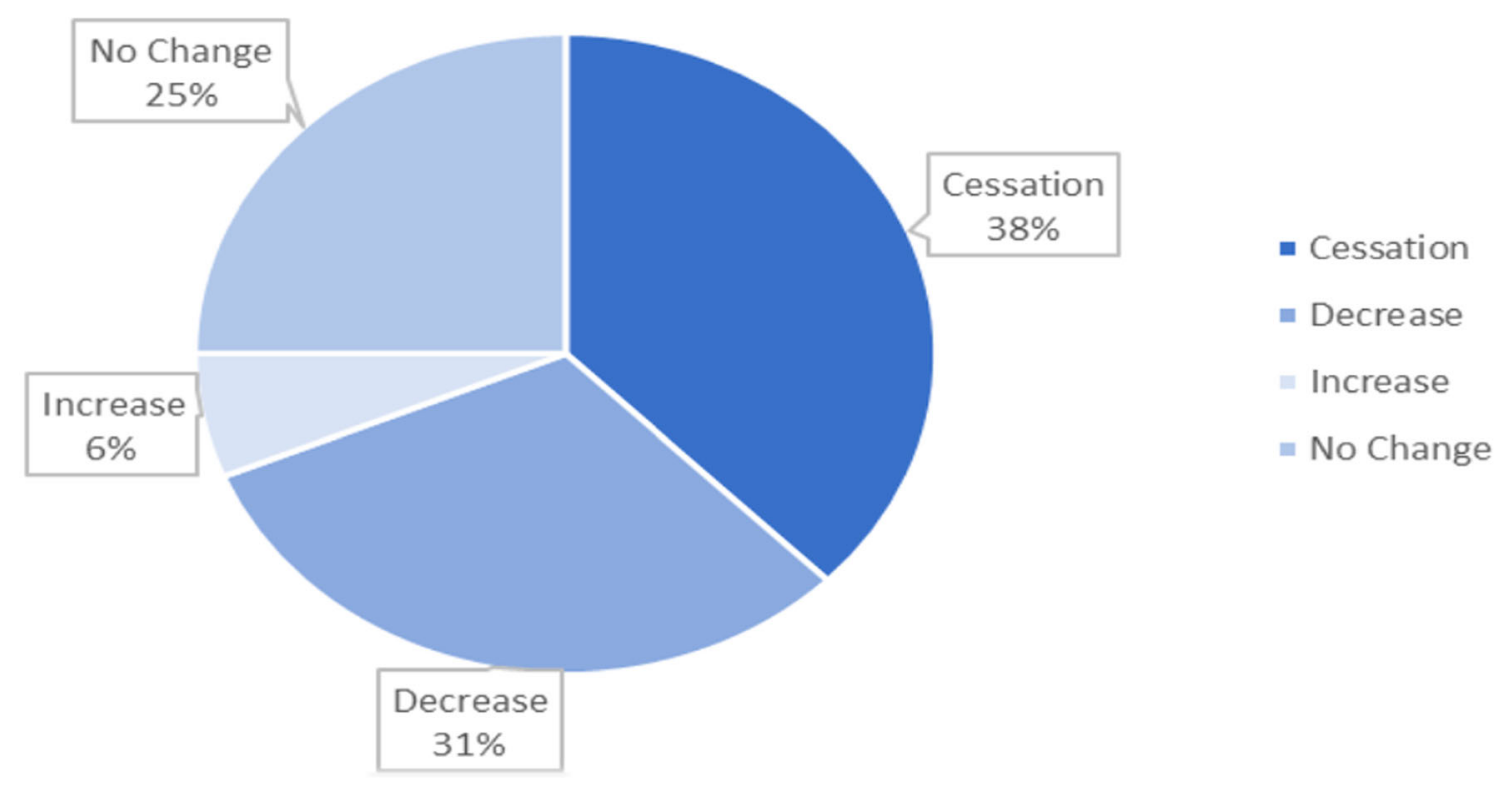

Fig. 3 Change in opioid therapy as a result of virtual reality therapy for 12 weeks. Percentage of patients with either no change, increase, decrease, or complete cessation are depicted

\section{Goal Improvements}

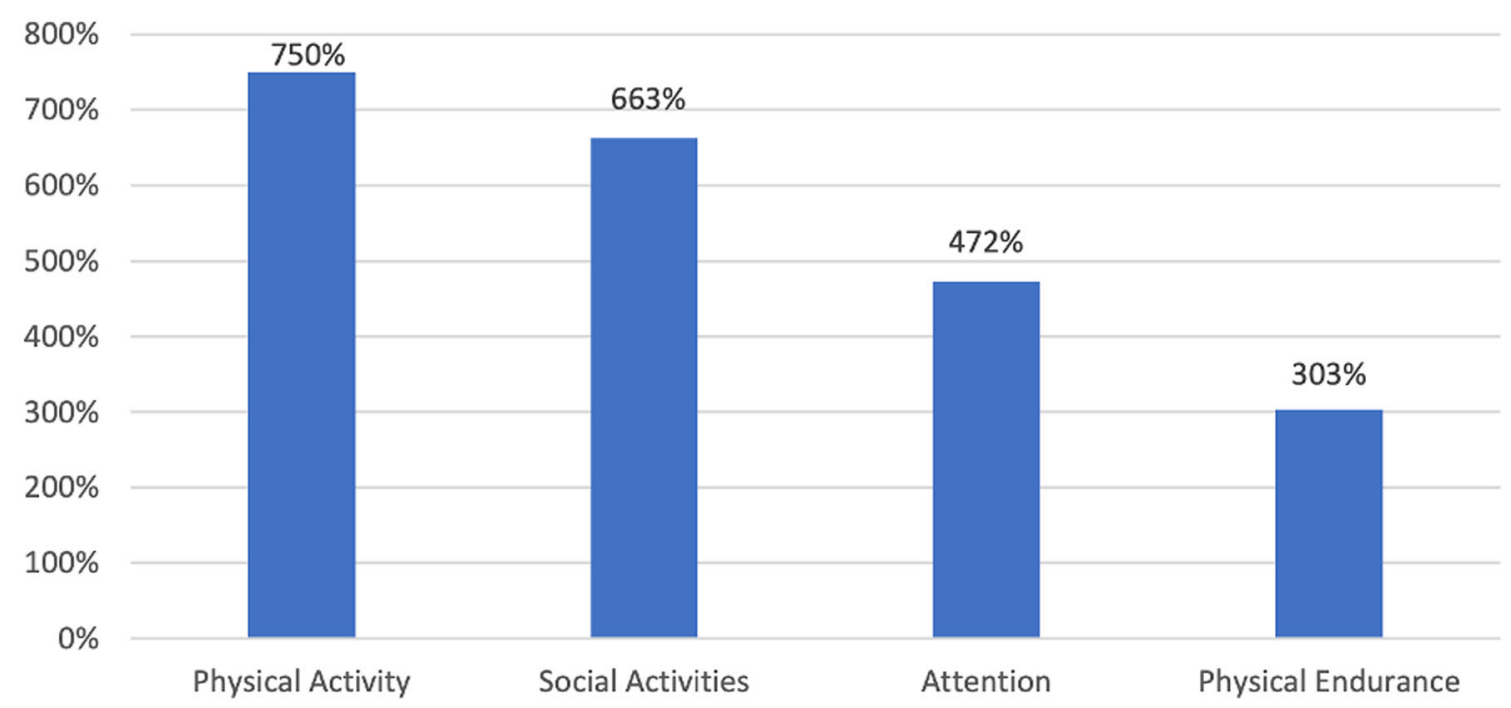

Fig. 4 Improvement in patient goals as a result of virtual reality therapy after 12 weeks. Percentage of improvement towards goal from baseline (prior to initiation of therapy) is depicted 


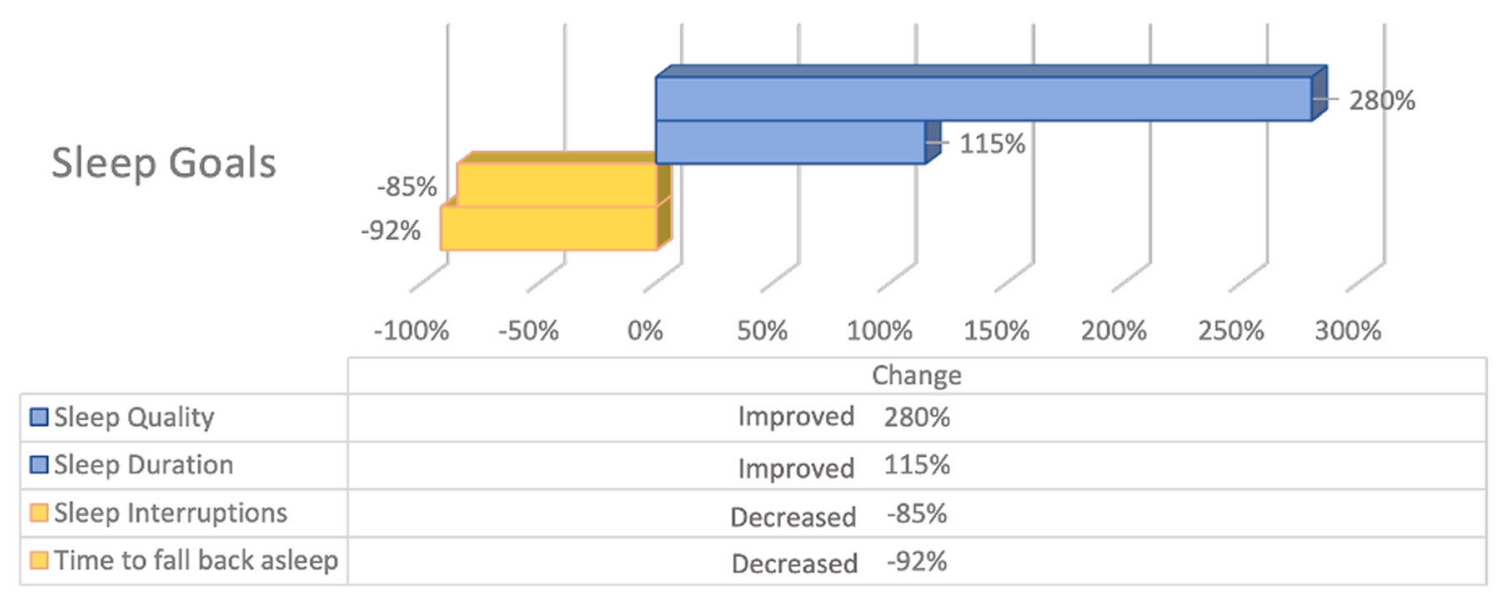

Fig. 5 Percentage change in sleep goals for patients after completion of 12 weeks of virtual reality therapy. Percentage of improvement towards goal from baseline (prior to initiation of therapy) is depicted

\section{Behavioral and Physical Health}

After completing the 12-week program, patients reported an approximately $470 \%$ increase in their ability to focus and complete tasks. Further, patients reported a 70\% reduction in anxiety related to pain, thereby leading to increased engagement in their specific behavioral activity goals.

In regard to physical activity specifically, many patients reported considerable difficulty in performing physical activities secondary to limitations of pain. However, after completion of the program, patients reported a near 300\% increase in the duration of time physical activities could be performed and a seven-times increase in the number of activities performed.

\section{Social Well-Being}

Prior to initiating therapy, the majority of patients reported that pain and psycho-social comorbidities significantly interfered with their participation in social activities. However, after completion of the 12-week program, patients reported an overall seven-times increase in their participation in social activities. Furthermore, patients reported an $85 \%$ decrease in overall forgetfulness and $57 \%$ decrease in fatigue.

\section{Patient Immersion and Satisfaction}

Across all 12-weeks of the programs, patients appeared to be highly immersed and satisfied with virtual reality therapy. Importantly, near the end of the program, on average, patients reported their satisfaction and immersion at a mean of 8.0.

\section{DISCUSSION}

The overarching results of our retrospective analysis suggests that the Harvard MedTech Vx Pain Relief Program can be an effective means to manage acute and chronic pain related to workrelated injuries. The program, when completed, appears to provide meaningful reductions in pain and opioid use, while also improving the psychosocial aspects of pain, such as sleep, behavior, and physical activity. Importantly, the program was well received by patients, and very little difficulty was noted immersing in virtual reality therapy. Taken together, our findings support the potential role that virtual reality therapy, through the Harvard MedTech Vx Pain Relief Program, can play a part of a multimodal therapeutic option for treating patients with acute and chronic pain.

Pain is both a sensory and an emotional experience and is also known to be affected by various psychological factors [5]. Several different theories of pain control have been proposed, however, one of the most widely adopted theories revolves around the Gage Control Theory of pain, which posits that alternative stimuli can reduce the intensity of 
physical pain by blocking pain messages at nerve gates in the spinal cord [6]. Virtual reality therapy has the ability to affect this pathway by blocking or de-sensitizing the physiologic sensation of pain from the peripheral nervous system through psychological inputs to the brain [4]. Mechanisms of this control on an acute level stem from the possible analgesic role of virtual reality, which may be due to intercortical modulation of pain-related signals such as touch and memory [7]. Indeed with much of the science research focused on its role in the acute setting, clinical evidence has thus far found benefit of its use in the acute pain population, such as in burn care patients and outpatient medical procedures $[8,9]$. However, our evidence sheds insight on its potential beneficial role for chronic pain patients, and especially those who have work-related disabilities. Further, the results of our review further suggest that improvements in both the physical aspects of pain, in addition to the psychosomatic aspects/behavioral components, lend way to sustained positive outcomes for patients.

For the chronic pain population, our evidence is largely consistent with other smaller studies [10-12] that have investigated the use of virtual reality. However, with a much larger patient population, our study was able to demonstrate that the program can be successfully adhered to and incorporated as a part of a multimodal regimen to treat patients. This becomes especially important in the light of the COVID-19 pandemic, which has prompted a shift towards telemedicine in treating patients [13]. Equally as important, virtual reality may offer patients a remote option for treatment of their pain that could easily supplement existing treatments. The role of virtual reality therapy in this setting may become integral in further neuromodulation techniques that can be employed by the chronic pain physician.

It is also important to highlight aspects of the therapy program itself. Although Harvard MedTech Vx Pain Relief Program lasted 12 weeks, the optimal duration of therapy and number of sessions needed has yet to be fully established. For instance, in case [12] where virtual reality was used to help treat chronic neuropathic pain, a total of 33 sessions were used to provide sustained pain relief. In contrast, a study conducted on burn patients requiring multiple treatments used as little as three virtual reality sessions on separate days have also been used [11]. Based on our findings and evidence from prior studies, it appears that the optimal duration of therapy may vary based on the pain state, type, and patient characteristics. Further, there is some evidence that repeated exposure to virtual reality therapy may lead to desensitization; however, our analysis and those by Rutter et al. (2009) [14] appear to contradict this claim since benefits of virtual reality therapy were maintained and persisted.

\section{Strengths and Limitations}

Our analysis comes with several strengths. In one, by using a work-related disability population, we covered a select group of patients that were likely to suffer from chronic pain as a result of their injury. Further, restricting our focus to a homogenous population (i.e., workplace disability) enabled us to validate the therapeutic benefits of virtual reality therapy for this specific group of patients. Second, all patients were able to complete the Harvard MedTech Vx Pain Relief Program. This is very encouraging and indicates that this therapy may be considered practical and not cumbersome for patients to use. Third, we were uniquely able to identify reductions in pain and opioid use as a result of therapy implementation. Finally, even though the resource limited COVID-19 pandemic, we were able to implement the virtual training therapy program, which signifies its ease of use and limited barriers to adherence.

Our study also comes with several limitations. First, we did not include a control group of patients who did not undergo the intervention. Unfortunately, as a result of this, we are unable to draw conclusions regarding the true incremental effectiveness of the Harvard MedTech Vx Pain Relief Program in comparison to standard behavioral therapy. Second, our results were limited by the lack of quantification of opioid reduction; we were able to provide qualitative data on this outcome, however, 
quantitative data may be more meaningful for the clinician. Third, we were unable to evaluate the cost of using such an intervention for patients. Given that electronic innovations can be cost prohibitive, this is an aspect that needs to be investigated prior to widespread use of the program. Fourth, our analysis was limited to workplace injury patients; however, pain may be perceived differently by this population $[15,16]$, and this limits the external generalizability of our results.

\section{CONCLUSIONS}

In conclusion, the Harvard MedTech Vx Therapy appears to be an effective tool in the treatment of pain and the psycho-social issues associated with pain. This translates into a greater degree of pain resiliency and an overall improvement in general wellness and improved workplace functionality. Overall, the therapy appears to provide another effective option in managing acute and chronic pain in patients with work-related injuries using a multimodal approach.

\section{ACKNOWLEDGEMENTS}

Funding. No funding or sponsorship was received for this study or publication of this article.

Authorship. All named authors meet the International Committee of Medical Journal Editors (ICMJE) criteria for authorship for this article, take responsibility for the integrity of the work as a whole, and have given their approval for this version to be published.

Authorship Contributions. Alaa Abd-Elsayed, MD. Study design, literature review, writing the article, and editing.

Nasir Hussain, MD. Literature review, writing the article, and editing.

Gerry Stanley, MD. Study design, writing article.
Disclosures. Alaa Abd-Elsayed is a consultant of Medtronic, Harvard MedTech and Avanos. Gerry Stanley holds a leadership position at Harvard MedTech. Nasir Hussain has nothing to disclose.

Compliance with Ethics Guidelines. In the setting of the COVID-19 pandemic, this retrospective cohort study received institutional review board (IRB) exempt status due to investigation of a benign behavioral intervention. This study was performed in accordance with the Helsinki Declaration of 1964 and its later amendments.

Open Access. This article is licensed under a Creative Commons Attribution-NonCommercial 4.0 International License, which permits any non-commercial use, sharing, adaptation, distribution and reproduction in any medium or format, as long as you give appropriate credit to the original author(s) and the source, provide a link to the Creative Commons licence, and indicate if changes were made. The images or other third party material in this article are included in the article's Creative Commons licence, unless indicated otherwise in a credit line to the material. If material is not included in the article's Creative Commons licence and your intended use is not permitted by statutory regulation or exceeds the permitted use, you will need to obtain permission directly from the copyright holder. To view a copy of this licence, visit http://creativecommons.org/licenses/by$\mathrm{nc} / 4.0 /$.

\section{REFERENCES}

1. Zulman DM, Verghese A. Virtual Care, telemedicine visits, and real connection in the era of COVID-19: unforeseen opportunity in the face of adversity. JAMA. 2021;325:437-8.

2. Linton SJ, Shaw WS. Impact of psychological factors in the experience of pain. Phys Ther. 2011;91: 700-11.

3. Rosen CS, Morland LA, Glassman LH, Marx BP, Weaver K, Smith CA, Pollack S, Schnurr PP. Virtual mental health care in the Veterans Health 
Administration's immediate response to coronavirus disease-19. Am Psychol. 2021;76:26-38.

4. Li A, Montaño Z, Chen VJ, Gold JI. Virtual reality and pain management: current trends and future directions. Pain Manag. 2011;1:147-57.

5. Reddi D, Curran N, Stephens R. An introduction to pain pathways and mechanisms. Br J Hosp Med (Lond). 2013;74(Suppl 12):C188-91.

6. Katz J, Rosenbloom BN. The golden anniversary of Melzack and Wall's gate control theory of pain: celebrating 50 years of pain research and management. Pain Res Manag. 2015;20:285-6.

7. Gold JI, Belmont KA, Thomas DA. The neurobiology of virtual reality pain attenuation. Cyberpsychol Behav. 2007;10:536-44.

8. Gold J, Reger G, Rizzo A, Buckwalter G, Kim S, Joseph M. Virtual reality in outpatient phlebotomy: evaluating pediatric pain distraction during blood draw. J Pain. 2005;6:S57.

9. Hoffman HG, Patterson DR, Seibel E, Soltani M, Jewett-Leahy L, Sharar SR. Virtual reality pain control during burn wound debridement in the hydrotank. Clin J Pain. 2008;24:299-304.

10. Sato K, Fukumori S, Matsusaki T, Maruo T, Ishikawa S, Nishie H, Takata K, Mizuhara H, Mizobuchi S, Nakatsuka H, Matsumi M, Gofuku A, Yokoyama M,
Morita K. Nonimmersive virtual reality mirror visual feedback therapy and its application for the treatment of complex regional pain syndrome: an open-label pilot study. Pain Med. 2010;11:622-9.

11. Hoffman HG, Patterson DR, Carrougher GJ, Sharar SR. Effectiveness of virtual reality-based pain control with multiple treatments. Clin J Pain. 2001;17: 229-35.

12. Oneal BJ, Patterson DR, Soltani M, Teeley A, Jensen MP. Virtual reality hypnosis in the treatment of chronic neuropathic pain: a case report. Int J Clin Exp Hypn. 2008;56:451-62.

13. Monaghesh E, Hajizadeh A. The role of telehealth during COVID-19 outbreak: a systematic review based on current evidence. BMC Public Health. 2020;20:1193.

14. Rutter CE, Dahlquist LM, Weiss KE. Sustained efficacy of virtual reality distraction. J Pain. 2009;10: 391-7.

15. Vowles KE, Gross RT. Work-related beliefs about injury and physical capability for work in individuals with chronic pain. Pain. 2003;101:291-8.

16. Phillips LA, Carroll LJ, Voaklander DC, Gross DP, Beach JR. Pain coping in injured workers with chronic pain: what's unique about workers? Disabil Rehabil. 2012;34:1774-82. 